\title{
Tempo Discrimination in 3- and 4-year-old children: performances and threshold
}

Anne Bobin-Bègue and Joëlle Provasi

\section{(2) OpenEdition}

1 Journals

Electronic version

URL: http://journals.openedition.org/cpl/440

DOI: $10.4000 / \mathrm{cpl} .440$

ISSN: $1379-6100$

Publisher

Centre PsyCLÉ

\section{Electronic reference}

Anne Bobin-Bègue and Joëlle Provasi, «Tempo Discrimination in 3- and 4-year-old children: performances and threshold », Current psychology letters [Online], 16, Vol. 2, 2005 | 2005, Online since 04 May 2005, connection on 08 September 2020. URL : http://journals.openedition.org/cpl/440 ; DOI : https://doi.org/10.4000/cpl.440

This text was automatically generated on 8 September 2020

(C) All rights reserved 


\title{
Tempo Discrimination in 3- and 4- year-old children: performances and threshold
}

\author{
Anne Bobin-Bègue and Joëlle Provasi
}

Received July 6, 2004

Revised March 19, 2005

Accepted April 25, 2005

On line May 4, 2005

The central nervous system must continuously analyze and process temporal information to interact adequately with the environment. The complex nature and the multiplicity of time scales make the associated cognitive processes both interesting and difficult to understand. Early childhood is a particularly important time for the study of short durations (i.e. durations of less than one second). Rhythmic behavioral activities such as sucking, kicking and crawling are associated, from the moment of birth, with particular stages of neuromuscular maturation. This temporary behavior, involving non-coordinated movements, eventually changes to voluntary and coordinated motor behavior (Thelen 1979). Temporal contingency is also of prime importance in intermodal perception (Schmuckler, 1996; Lewkowicz, 2000, 2003). Therefore, the developmental pattern in the temporal processing of young children is an interesting question.

Short duration processes are studied using an isochronous sequence of intervals. The frequency of these intervals is usually called the tempo ${ }^{1}$. Results show that when the number of intervals in a sequence is increased a specific interval can be more precisely processed, and that this is related to the amount of information available (Drake \& Botte, 1993). That is, the more intervals produced, the more precise is its memory trace and the greater the sensitivity. The accuracy of interval processing can be assessed by distinguishing one tempo from another (i.e. a discrimination task). Thus, tempo discrimination tasks and, more specifically, the discrimination threshold (i.e. the 
lowest perceived difference between two tempi) are important for modelling the psychophysical parameters of interval processing.

The theoretical framework for tempo discrimination is not yet clearly defined, with no notable time model accounting for tempo discrimination (see for example Wing \& Kristofferson, 1973a, 1973b; Church, 1984, Ivry \& Richardson, 2002). Even during childhood, only the Dynamic Attending Theory (Jones, 1976; Jones \& Boltz, 1989) is able to explain tempo discrimination. This psychological model was developed to explain, dynamically, how listeners focus their attention on a complex sequence of intervals. The listener's attention was found to be cyclic and appeared to have a biological origin. This model has been extended and it is now possible to make predictions about interval processing during early childhood.

In the Dynamic Attending Theory model, when listening to a rhythmic sequence, the listener's attention spontaneously focuses on events occurring at a specific tempo. This tempo is called the referent period and relates to a main single oscillator. The referent period is specific to each individual. Interval processing is more accurate for intervals close to the referent period than for intervals far from this value. Thus, the smallest perceived difference between two tempi describes a U-shaped function centered on the standard tempo being tested. Usually, for the discrimination task the subjects have to detect the fastest tempo between the standard tempo and a variable one.

In the childhood extended model, Drake, Jones and Baruch (2000) hypothesized that the referent period slows down with age. According to Fraisse (1974) and Drake et al. (2000), the referent period could be determined by measuring the Spontaneous Motor Tempo (SMT). The SMT is a manual tapping task at the most comfortable tempo and is around $400 \mathrm{~ms}$ between the ages of $2^{1 / 2}$ and $4 \frac{1}{2}$ years (Gérard and Rosenfeld, 1995; Provasi \& Bobin-Bègue, 2003), around $500 \mathrm{~ms}$ for a 10 year-old (Drake et al., 2000), around $600 \mathrm{~ms}$ for adults (Fraisse, 1974; Collyer, Broadbent \& Church, 1992) and around $750 \mathrm{~ms}$ for elderly people (Vanneste, Pouthas \& Wearden, 2001).

This model also states that, with age, the development of focal attending is related to the passage from the initial use of a single oscillator, underlying the referent period, towards the coupling of multiple oscillators. Thus, the range of accessible tempi extends with age. Drake et al. (2000) observed an increase in the range of tempi at which subjects can perform temporal tasks (such as the SMT or tempo discrimination tasks). This describes a larger U-shaped function of discrimination performance with respect to the tempi tested.

Therefore, in a discrimination task, because a child's referent period is faster than an adult's, the best performances for children are seen at faster tempi (i.e. shorter durations) than for adults. This was shown by experimental data obtained from children over 4 years of age. However, due to the lack of psychophysical data from children under 4 years of age, it is not known whether this model is valid from birth. It is therefore important to determine whether these developmental rules applied to children under 4 years of age or whether they are completely different due to the rapid neural development during the first years of life.

The main reason for the lack of evidence during early childhood is the difficulty of obtaining reliable experimental data with very young children. The only way to collect reliable data is to perform repetitive experiments that are unappealing for young subjects. Therefore, procedures should be adapted to children's psychomotor skills and to the level of attention they are able to devote to the task. As the psychomotor and 
cognitive skills of young children evolve quickly, often within a few months or even weeks, experimental protocols must be designed for a few months at the most. Therefore the study of time perception and tempo discrimination in subjects less than 4 years of age requires the development of specific procedures designed for such a narrow age range.

Available data

Extremely repetitive discrimination procedures have established that, for a standard tempo at the referent period, the discrimination threshold corresponds to a difference of $3 \%$ in untrained adults and of $1.6 \%$ for adults trained for discrimination tasks. That is, at best an adult can discriminate a $590 \mathrm{~ms}$ tempo from a $600 \mathrm{~ms}$ one (Drake \& Botte, 1993). However, the few studies with children, have shown only a $15 \%$ difference between the standard tempo and the variable one (Baruch \& Drake, 1997; Drake et al., 2000). In fact, no discrimination threshold value was established, even at the referent period, since the referent period has not yet been clearly defined in children.

Child subjects over 4 years of age were required to differentiate a standard tempo from one $15 \%$ faster (Drake \& Baruch, 1995). Four-year-old children could discriminate a $300 \mathrm{~ms}$ tempo from a tempo $15 \%$ faster, but not for the other tempi tested $(100,600$, 1000 and $1500 \mathrm{~ms}$ ). Although this does not allow the determination the optimal threshold, it shows the optimal sensitivity at 4 years should be around $300 \mathrm{~ms}$.

Conflicting data have been collected on tempo discrimination during infancy. Infants are able to modify the temporal pattern of their rhythmic behavior depending on external stimulation (Provasi, 1988; Pouthas, Provasi \& Droit, 1996; Chang \& Trehub, 1977) and are able to discriminate 2 different rhythms (Demany, McKenzie \& Vurpillot, 1977). Baruch and Drake (1997) found that, with habituation, 2 and 4-month-old infants are able to discriminate a $600 \mathrm{~ms}$ tempo from one $15 \%$ faster (i.e. $510 \mathrm{~ms}$ ). However, this was not seen for other tested tempi $(100,300$ and $1500 \mathrm{~ms})$ or for any tempo slower (15\% slower) than that used for habituation. Also, a discrimination threshold was not determined for infants in this experiment. This suggests that the tempo of optimal sensitivity should be close to $600 \mathrm{~ms}$. However, this disagrees with the performance of 4 -year-olds (discrimination of $15 \%$ faster only at $300 \mathrm{~ms}$ tempo, Drake \& Baruch, 1995), even if, at this age, the discrimination threshold at $600 \mathrm{~ms}$ should be higher than $15 \%$. According to the developmental model, the optimal threshold is observed for the referent period. However, results suggest that at 4 years of age, the referent period is closer to $300 \mathrm{~ms}$ (and probably around $400 \mathrm{~ms}$ according to Gérard and Rosenfeld, 1995; Provasi \& Bobin-Bègue, 2003) whereas in infants, it is closer to $600 \mathrm{~ms}$. This is the same tempo as seen in adults, suggesting the referent period does not slow down, at least during early childhood. The features of the developmental model, especially the Ushaped function of discrimination performance, probably do not apply to children under 4 years of age. Therefore, further research is needed from two perspectives. The first is to study the discrimination thresholds over a wide range of tempi to determine the optimal tempo sensitivity, and consequently the referent period, for a particular age. The second is developmental: to compare discrimination performances and thresholds for a particular tempo, at different ages. In our study, we chose to explore tempo discrimination during the first years of life, as the referent period is not yet clearly defined at this age. From a developmental context our results should give concrete values for discrimination thresholds and optimal tempo sensitivities. More practically, it will contribute to the development of experimental paradigms adapted to young children. Most experiments have shown that the best discrimination 
performances were observed for intervals around $600 \mathrm{~ms}$ both for infants and adults. Therefore, using a suitable paradigm, $600 \mathrm{~ms}$ is an appropriate value for exploring young children's tempo discrimination performances.

Issues and hypotheses

The aim of our experiment is to estimate the discrimination threshold and performance for the specific tempo of $600 \mathrm{~ms}$ in children of less than 4 years of age.

We have concentrated on children between 3 and 4 years of age for 4 reasons: first, time estimation improves considerably during this period; second, no psychophysical data are available at 3 years of age; third, childhood data are conflicting with regard to the model; and finally, it allows us to compare our results with those from studies of children over 4 years of age. From a methodological point of view, as the protocol must contain specific age-related constraints, a given procedure is only suitable for an age range of only a few months.

There have been few studies concerning young children, as timing tasks carried out with adults require very long and unappealing procedures needing sustained attention. With young children, procedures must not only provide reliable data (i.e. many trials) but also must not be too tedious (e.g. an intra-session comparison must be done between successive sets of trials in order to control for boredom).

To explore the tempo discrimination performance at these young ages, our experimental design used 2 variable tempi either side of $600 \mathrm{~ms}$ (one couple of tempi per session). This allowed us to remain at this intermediate tempo and also avoided a training effect on a standard tempo. However, it has the disadvantage of making the results of our study difficult to compare directly with the literature. Other experiments tested only a $15 \%$ difference between a standard tempo and a variable one. The aim of our study was to design a protocol, suitable for young children, to approximate the discrimination threshold at a young age. During each session, after a training set, the child had to identify whether the tempo heard was a slow or fast one. Throughout 5 sessions, the difference between the 2 tempi decreased: the difficulty of discriminating the 2 tempi increased. This method allowed us to specify discrimination performances at $600 \mathrm{~ms}$ for 3 and 4 year-old children. We aimed to determine the discrimination threshold for this tempo. It should be around 15\%, as the discrimination threshold is less than or equal to $15 \%$ in infants (Baruch \& Drake, 1997) and above $15 \%$ at 4 years of age (Drake \& Baruch, 1995). We expected to find a decrease in discrimination performances with increasing difficulty of the test and an improvement in performance between the ages of 3 and 4 years.

MethodParticipants

Forty-eight children took part in the experiment: twenty-six 4-year-olds (8 girls and 18 boys; mean age: 3 years $9 \frac{1}{2}$ months, $S D=22$ days, range: 3 years $8 \frac{1}{2}$ months -3 years $11^{1 / 2}$ months) and twenty-two 3 -year-olds ( 10 girls and 12 boys; mean age $=3$ years $2 \frac{1}{2}$ months, $\mathrm{SD}=22$ days, range: 3 years 2 months -3 years 4 months). Nine additional children were tested but were excluded from data analysis because they did not want to play the game. They were all recruited from the same nursery school in Paris (France), and were from the same class level. Their parents had previously given their written and informed consent concerning the experimental procedure.

\section{Apparatus}

A portable computer was connected to a 2-button ( $4 \mathrm{~cm}$ diameter buttons) wooden box and to a speaker. Each button was sufficiently spread out $(20 \mathrm{~cm}$ between the 2 buttons) 
so as to be pressed only by the corresponding hand - left button, left hand; right button, right hand. Two pictures, representing a rabbit and a tortoise with the same features (size, colors, drawing etc), were placed beside each button, with the rabbit on the left button and the tortoise on the right button. Each button remained unchanged throughout the entire experiment to allow the child to learn.

A computer screen ideally oriented for the child was used to deliver the reinforcement stimulus (200 pixels $-10 \mathrm{~cm} \times 10 \mathrm{~cm}$ ).

Stimuli

The computer generated a trial, defined as a sequence of 10 -tones $(50 \mathrm{~ms}, 1000 \mathrm{~Hz})$ at a fixed Inter Stimulus Interval (ISI). A set was composed of 10 trials at a fast ISI and 10 trials at a slow ISI. The fast and slow ISI were either side of a $600 \mathrm{~ms}$ ISI, denoted 策 X\%. Within each set the order of the 2 ISIs were randomly generated by the computer.

The correct response for the fast ISI was to press the left button (rabbit). A reinforcement stimulus of a picture of the rabbit appeared on the screen immediately after the correct response. For the slow ISI, the correct response of pressing the right button resulted in a picture of the tortoise appearing on the computer screen. The responses had to be given within a 5-second period following the end of the trial. After a correct response the reinforcement stimulus (the ISI associated picture) appeared on the screen for 1 second. No response or a false response resulted in nothing appearing on the screen. The computer recorded the response (right press, left press or none) given for each trial. We logged the number of correct responses per set. The beginning of the following trial was triggered by the experimenter to ensure that the child was paying attention to the sound.

Procedure

The experiment took place in an isolated room, next to a classroom. The child, alone with the experimenter, was seated in a suitable chair in front of a table, on which the computer was placed.

Our experiment was divided into 5 sessions: one per day. The period between the first and the fifth session was about 11 days (mean $=10,94$ days, $\mathrm{SD}=4,24$ days). Each session (lasting about 15 minutes) measured the child's responses to the fast and slow ISI. The 2 tempi tested in each session varied from a tempo of $600 \mathrm{~ms}$ ISI in the following order:

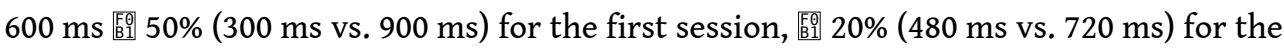

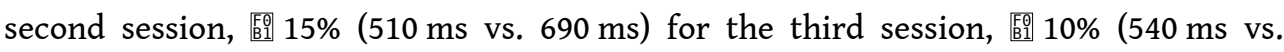
$660 \mathrm{~ms}$ ) for the fourth session, and 原 $5 \%$ (570 ms vs. $630 \mathrm{~ms}$ ) for the last session. The first session was designed to be very easy, to prove that the task could be performed by children so young. The session order was chosen to be become progressively more difficult. There are 2 reasons to justify this choice. First, usual adult discrimination procedures are carried out in the same way: each time the subject succeeds, the task becomes progressively more difficult (Bonnet, 1986). Second, to ensure that the children could manage the task, even at a difficult level, we increased the difficulty throughout the 5 sessions. The only learning effect that could be caused by the session order was the procedure. As the 2 tempi tested were changed for each session this allowed the acquisition of reliable data. The limited number of trials for such young children (60 for each tempo) compared to adults procedures (and even with infant habituation procedures), allows us to believe that no 
training effects should be observed. Consequently, the session order was deliberately not counterbalanced.

Each session was composed of a demonstration and then 3 sets of 20 trials (see Table 1). The last 2 sets were designed to confirm the absence of any training effects.

Table 1: Session organization

\begin{tabular}{|c|c|c|c|}
\hline Session characteristics & Sets & Conditions & $\begin{array}{l}\text { Set composition } \\
\text { (trials* randomly mixed) }\end{array}$ \\
\hline \multirow{4}{*}{ Session: $600 \mathrm{~ms} \pm \mathrm{X} \%$} & Demonstration & $\begin{array}{l}\text { "Listen to the rabbit" } \\
\text { "Listen to the tortoise" }\end{array}$ & $\begin{array}{l}1 \text { trial at the fast IOI } \\
\text { I trial at the slow IOI }\end{array}$ \\
\hline & Set 1 & Assisted & $\begin{array}{l}10 \text { trials at the fast } 101 \\
10 \text { trials at the slow IOI }\end{array}$ \\
\hline & Set 2 & Without assistance & $\begin{array}{l}10 \text { trials at the fast } 101 \\
10 \text { trials at the slow } 101\end{array}$ \\
\hline & Set 3 & Without assistance & $\begin{array}{l}10 \text { trials at the fast } 101 \\
10 \text { trials at the slow } 101\end{array}$ \\
\hline
\end{tabular}

*A trial is a 10-tone isochronous sequence at a specific IOI.

Fast IOI $=600 \mathrm{~ms} \times(1-\mathrm{X})$

Slow IOI $=600 \mathrm{~ms} \times(1+\mathrm{X})$

During the demonstration, the only words said by the experimenter were, "Listen to the sound of the rabbit/tortoise" and "So, press the rabbit/tortoise's button and look." Before the first set, the experimenter said, "First I will help you, and then you will play alone." The first set was intended to make the association between the fast/slow ISI and the corresponding animal picture. This was done with the experimenter's help and verbal feedback (for example: "look the rabbit/tortoise" or "it was not the rabbit/ tortoise's sound"). In the following 2 sets the child played alone with no outside help or verbal instruction. A comparison with the last 2 sets of each session allowed us to assess a potential learning or boredom effect. A preferred-side effect could be detected by the number of correct responses (about 50\%).

Results

A trial score of 1 was allocated for a correct response per trial. For each set, correct responses were counted, giving set scores ranging from 0 to 20. Chi-squared tests for all subjects showed whether responses for each set were different from random responses. Analyses of variance (ANOVA) tests were then carried out for two between-subject factors (sex and age) and two within-subject factors (session and set).

We compared in each set, and for all subjects, the number of correct responses obtained with the theoretical random result of 10 , to prove that children discriminated the two ISIs. Chi-squared tests showed that children did not give random responses, except for the last session. These results are strengthened by the comparison with a normal distribution of random responses (mean of 10 and a standard deviation of 2; Kolmogorov-Smirnov's test) (see Table 2). 
Table 2: Results of analyses testing randomized responses for each session and for each age group

\begin{tabular}{|c|c|c|c|c|c|c|c|}
\hline \multirow[t]{2}{*}{ Session } & \multirow[t]{2}{*}{$\begin{array}{c}\text { Age } \\
\text { (years) }\end{array}$} & \multirow[t]{2}{*}{$\begin{array}{l}\text { Number } \\
\text { of } \\
\text { subjects }\end{array}$} & \multirow[t]{2}{*}{$\begin{array}{l}\text { Session } \\
\text { Score } \\
\text { Mean }\end{array}$} & \multicolumn{2}{|c|}{$\begin{array}{c}\text { Comparison } \\
\text { between session } \\
\text { score and random } \\
\text { score }(10)\end{array}$} & \multicolumn{2}{|c|}{$\begin{array}{c}\text { Adjustement with } \\
\text { n(10:4) } \\
\text { Kolmogorov- } \\
\text { Smirnov }\end{array}$} \\
\hline & & & & Chi_ & $p<$ & d & $p<$ \\
\hline \multirow{2}{*}{1} & 3 & 22 & 14.25 & 84.43 & .001 & .524 & .01 \\
\hline & 4 & 25 & 17.14 & 151.73 & .001 & .754 & .01 \\
\hline \multirow{2}{*}{2} & 3 & 20 & 12.78 & 62.53 & .001 & .477 & .01 \\
\hline & 4 & 21 & 16.29 & 103.00 & .001 & .790 & .01 \\
\hline \multirow{2}{*}{3} & 3 & 17 & 11.29 & 32.60 & .01 & 339 & .05 \\
\hline & 4 & 22 & 14.61 & 85.38 & .001 & .615 & .01 \\
\hline \multirow{2}{*}{4} & 3 & 17 & 11.82 & 24.60 & NS & .229 & NS \\
\hline & 4 & 20 & 13.68 & 52.28 & .001 & .477 & .01 \\
\hline \multirow{2}{*}{5} & 3 & 16 & 9.31 & 4.85 & NS & .246 & NS \\
\hline & 4 & 19 & 10.53 & 7.68 & NS & .237 & NS \\
\hline
\end{tabular}

A Fisher test on the total number of right and left button presses showed that the idea of a preferred-side effect had to be rejected (p>.05) as no preferred-hand bias was observed.

ANOVA was performed on the number of correct responses, revealing two principle effects. The first was an age effect, $F(1,28)=5.12 ; p<.05$ : the older children performed better than the younger children.

The second, was a session effect, $F(4,112)=27.33 ; p<.001$ : the scores decreased across sessions. As expected, discrimination performance decreased with increasing task difficulty. Children obtained similar scores during the first and the second session (Tukey's post-hoc tests: the first session differed from the third, $p<.0005$, from the fourth, $p<.0005$ and from the fifth, $p<.0005$, and the second session differed from the third, $p<.01$, from the fourth, $p<.05$ and from the fifth, $p<.0005$ ), the third and the fourth sessions were also statistically similar, whereas both differed from the fifth session (Tukey's post-hoc test, $p<.0005$ and $p<.0005$, respectively).

There were no effects due to sex and set, and no interaction between set and session factors. Performances during set 2 were similar to performances during set 3 , whatever the session. Consequently, within a given session, no learning or boredom effect could be seen.

Finally, there was an interaction between age and session factors, $F(4,112)=3.15 ; p<.05$, revealed by different decreases among sessions depending on age (see Figure 1). 


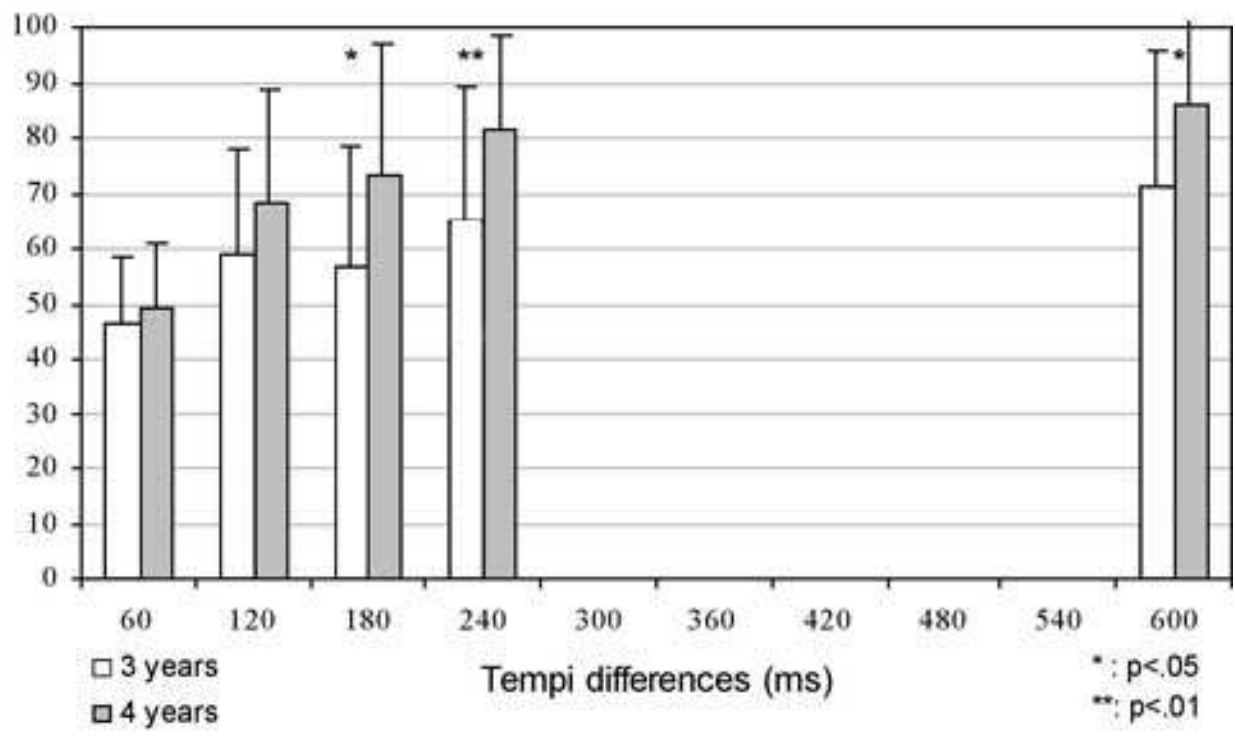

Image4Figure 1: Mean score for each session as a function of age

This interaction was analyzed using Tukey's post-hoc tests. Between the 2 age groups, the older children performed better than the younger children for only the first three sessions (respectively: $p<.001, p<.001$ and $p<.05$ ), with their scores being similar for the last two sessions (S4 and $\mathrm{S} 5$ ). The younger children's performances were similar for almost all the sessions. However, both session $1(p<.0005)$ and session $2(p<.05)$ differed from session 5. The older children's performances differed as a function of the session. The first session differed from the last three sessions (S1 vs. S3 $p<.005$, S1 vs. S4 $p<.005$, $\mathrm{S} 1$ vs. S5 $p<.0005$ ); the second session differed from the last two sessions (S2 vs. S4 $p<.05$, S2 vs. S5 $p<.0005)$ and the third and the fourth sessions differed from the last session (S3 vs. S5 $p<.0005$, S4 vs. S5 $p<.0005$ ). Finally, for the older children S1 and S2 were performed as well as $\mathrm{S} 3$ and $\mathrm{S} 4$.

Discussion

Our experiment was designed to assess performances in discrimination at intermediate tempi (about $600 \mathrm{~ms}$ ). According to certain studies this seems to be the optimal sensitivity zone in both children and adults. Two age groups (3- and 4-year-old) had to discriminate 2 tempi during 5 sessions: respectively $600 \mathrm{~ms} \pm 50 \%, \pm 20 \%, \pm 15 \%, \pm 10 \%$, $\pm 5 \%$. The principle results were: first, performance decreased with increasing difficulty, with the results in the last session being the same for purely random responses; second, a performance improvement was found in tempi discrimination from 3-year-olds to 4year-olds; third, 4-year-old children performed significantly better than the 3-year-olds during the first three sessions; and fourth, for the younger children in the fourth session their results were the same as for random responses whereas the older children could still discriminate the 2 tempi. That apparent random responses were not seen in the same session for the 2 age groups underlines the appropriate choice of five sessions.

Our procedure is clearly different from those typically used. When we refer to a $\pm \mathrm{X} \%$ difference, it means that the 2 tempi compared were $600-\mathrm{X} \% \mathrm{~ms}$ and $600+\mathrm{X} \% \mathrm{~ms}$. It could be argued that our slow tempo is similar to the usual "standard" tempo and the 
fast one to the "variable" one. However, as our slow tempo changes across sessions, it cannot be defined as a "standard" tempo. Consequently, we were careful to designate our tempo difference as " $\pm \mathrm{X} \%$." In usual discrimination procedures, the subject hears the 2 tempi successively and has to determine the faster one. However, our procedure was an identification task: the subject heard one of the 2 tempi and had to identify it as being either the faster or slower one. We chose this procedure because it allowed us to center the discrimination on $600 \mathrm{~ms}$, which is the normal reference value for perception and discrimination tasks. A difference of $\pm \mathrm{X} \%$ could approximately be reinterpreted as a "usual" difference of $2 \mathrm{X} \%$ when comparing our results to previous studies.

Discrimination performances

The number of correct responses decreased with increasing difficulty, ranging from $79 \%$ for the first session to $64 \%$ for the fourth. Discrimination performance is normally studied between a standard tempo and one $15 \%$ faster. In our experiment, 4-year-old children were able to discriminate a $540 \mathrm{~ms}$ tempo from a $660 \mathrm{~ms}$ tempo (the mean success rate differed significantly from random responses). However, the Drake and Baruch experiment (1995) showed that 4-year-old children could not discriminate a $600 \mathrm{~ms}$ tempo from a $510 \mathrm{~ms}$ tempo (the mean success rate did not differ significantly from random responses). This cannot be due to the ease of the task (an absolute difference of $90 \mathrm{~ms}$ vs. $120 \mathrm{~ms}$ ) but probably due to our procedure being designed for such young children. This may allow them to obtain better performances with our procedure than for a procedure designed for adults. The referent period for children less than 4 years old is about $430 \mathrm{~ms}$ (Provasi \& Bobin-Bègue, 2003). Therefore, 4-yearold children should be able to discriminate a $510 \mathrm{~ms}$ tempo from a $600 \mathrm{~ms}$ tempo, which is closer to the referent period than $540 \mathrm{~ms}$ vs. $660 \mathrm{~ms}$. This particular result and the fact that the children in our study were younger than the children in Drake's study suggests that our procedure comes closer to the real abilities of 3- and 4-year-old children.

Our results have also shown that at 4 years of age discrimination within the $\pm 50 \%$ to $\pm 20 \%$ ranges are equally easy. For an absolute difference between 240 and $600 \mathrm{~ms}$, the difficulty of discrimination is the same. This observation is also valid for discrimination within the $\pm 15 \%$ to $\pm 10 \%$ ranges (i.e. an absolute difference between 120 and $180 \mathrm{~ms}$ ), however discrimination performances were lower. These step-changes in difficulty were not seen for 3-year-old children. At this age, only the 2 easiest sessions had better scores than the most difficult session. We suggest that between 3 and 4 years of age, for tempi around $600 \mathrm{~ms}$, children's tempo discrimination improves by levels: easy level (above $\pm 20 \%$ difference in tempi), difficult level (between $\pm 10 \%$ to $\pm 20 \%$ ), and too difficult level (below $\pm 10 \%$ ). Our experiment studied timing abilities in the intermediate tempi range. From a developmental perspective, the experiment should be conducted on a wider range of tempi, especially around the referent period in young children of $430 \mathrm{~ms}$ (Provasi \& Bobin-Bègue, 2003).

Age-related performance

We found age-related differences in the discrimination performance for the first four sessions (where the responses were not given randomly). The older children performed significantly better than the younger children despite an age difference of only seven months. However, significant differences were only seen for the first three sessions. Step-changes in difficulty in the discrimination task were different between 3- and 4year-old children. This is in agreement with the improvement of rapid timing skills 
seen with age. Although our experiment was not designed to test this hypothesis, there could be a link between the development of timing abilities and the maturation of the cerebellum and basal ganglia, which is shown to be involved in the processing of relatively brief durations (Ivry \& Richardson, 2002; Clarke, Ivry, Grinband, Roberts \& Shimizu, 1996; Ivry \& Keele, 1989; Mattel \& Meck, 2000; Harrington, Haaland \& Hermanowicz, 1998; Gibbon, Malapani, Dale \& Gallistel, 1997). This may explain the improvement seen in short duration discrimination during these seven months. However, we cannot exclude the possibility of a lower level of attentiveness in the younger children. This is unlikely, though, as the age range in each group is very low and the between-subject variability of this general parameter was counterbalanced. Moreover, all the children were from the same classes and were given the same working activities. They participated in the experiment in the second part of the school year and were all stimulated in the same way for several months. Therefore we could suppose that the children's attention abilities were equivalent.

Discrimination threshold

Our study also allowed us to determine a range for the discrimination threshold for each age group. The 3-year-old children's threshold is situated between $\pm 15 \%$ and $\pm 10 \%$. The 4-year-old children were able to discriminate 2 tempi differing by up to $\pm 10 \%$, which is equivalent to a difference of $120 \mathrm{~ms}$ between the 2 tempi, but not tempi differing by $\pm 5 \%$ ( $60 \mathrm{~ms})$. We suggest the discrimination threshold is between $\pm 5 \%$ and $\pm 10 \%$ for $600 \mathrm{~ms}$ in 4-year-old children - a "usual" difference of between approximately $10 \%$ and $20 \%$. Although this experiment only gave discrimination threshold ranges it showed there was rapid improvement with age.

We can compare our data with previous studies. Our results for 4-year-old children are consistent with previous studies on 4-year-old children suggesting a threshold higher than $15 \%$, and with studies on infants showing that they can discriminate 2 tempi differing by $15 \%$ at $600 \mathrm{~ms}$ (Drake \& Baruch, 1995; Baruch \& Drake, 1997). These two studies suggest the threshold should be situated between $15 \%$ and $20 \%$ for 4 -year-old children and between $20 \%$ and $30 \%$ for 3-year-old children. This is far from the adults' $600 \mathrm{~ms}$ threshold of 1,6\% (Drake \& Botte, 1993). However, the adults' optimal sensitivity was obtained in specific conditions: only four adults participated and all were familiar with the psychoacoustic experiment. Therefore, this value should be considered as a special optimal value. In our experiment, the children were untrained and only familiar with the procedure. Our results suggest that, using our procedure, the discrimination threshold at $600 \mathrm{~ms}$ improves during young childhood. This has led us to believe the threshold value might be lower. As we only wanted to determine discrimination skills, we tested a wide range of values (from $\pm 50 \%$ to $\pm 5 \%$ ). Consequently, our procedure may not have a precise enough range for accurately determining the discrimination threshold. Also, 3 sessions allowed the majority of children to participate but led to less performance data and less accurate threshold values. For the last, fifth, session, children had participated 4 times in the experiment and already knew the procedure. They did not expect any new features in the game. A more precise threshold could be obtained after this period with only one additional session.

Conclusions and future research

This experiment provided for the first time the discrimination abilities of children between 3 and 4 years of age for short durations around $600 \mathrm{~ms}$. The results provide new, more accurate data and are consistent with those found for children above 4 years of age. The hypothesis of the improvement of timing accuracy, measured by the 
discrimination threshold, could be extended to children as young as 3-years old. These results probably do not reflect the overall abilities of children because this study focused on a tempo of $600 \mathrm{~ms}$. As optimal sensitivity is situated around the referent tempo (Drake et al., 2000) and as young children's referent tempo is about $430 \mathrm{~ms}$ (Provasi \& Bobin-Bègue, 2003), it could be argued that optimal sensitivity of children should be situated around $430 \mathrm{~ms}$. Other experiments need to be conducted at various tempi, and specifically at the child's referent period (Bobin-Bègue \& Provasi, submitted), to determine the U-shaped function of discrimination performance.

From a biological point of view there was a significant difference seen between the 2 age groups. During only 7 months timing abilities improve, which probably correlates with brain maturation. Finally, further studies are needed for younger children, especially between the ages of one and three, to determine the evolution of discrimination performance. A preliminary study was conducted with $2 \frac{1}{2}-$-year-old children, using the procedure described here but with adjusted parameters. It showed that this method is not appropriate for such young children. Thus, a new protocol should be designed for tempi discrimination in children less than 3 years of age, to confirm the U-shaped function postulated for short duration processing development.

This study has developed an experimental protocol for tempi discrimination for young children. It determined discrimination performances for 3- and 4-year-old children, which had not been tried before. Results showed that timing abilities, for short durations, evolve within a few months. Further experiments will use this protocol to determine the postulated U-shaped function expected at these ages. However, we should also investigate discrimination ability for younger children using an appropriately modified protocol (Bobin-Bègue \& Provasi, submitted).

Acknowledgements

The authors wish to thank Claude Kervella and Pierre Canet for their effective technical assistance and they extend their gratitude to the staff and children of the nursery school St Jacques (Paris, $5^{\text {th }}$ ) without whom this study could not have been carried out.

\section{BIBLIOGRAPHY}

Baruch, C., \& Drake, C. (1997). Tempo discrimination in infants. Infant Behavior and Development, 20 (4), 573-577.

Bonnet, C. (1986). Manuel pratique de Psychophysique. Paris: A; Colin.

Chang, H.-W., \& Thehub, S. E. (1977). Infant's perception of temporal grouping in auditory patterns. Child Development, 48, 1666-1670.

Church, R. M. (1984). Properties of the internal clock. In J. Gibbon \& L. Allan (Eds.), Timing and time perception (Vol. 423, pp. 566-582). New York: Academy of Sciences.

Clarke, S., Ivry, R., Grinband, J., Roberts, S., \& Shimizu, N. (1996). Exploring the domain of the cerebellar timing system: Elsevier Science B.V. 
Collyer, C. E., Broadbent, H. A., \& Church, R. M. (1992). Categorical time production: evidence for discrete timing in motor control. Perception \& Psychophysics, 51 No 2, 134-144.

Demany, L., McKenzie, B., \& Vurpillot, E. (1977). Rhythm perception in early infancy. Nature, 266 No5604, 718-719.

Drake, C., \& Botte, M.-C. (1993). Tempo sensitivity in auditory sequences : evidence for a multiplelook model. Perception \& Psychophysics, 54 (3), 277-286.

Drake, C., \& Baruch, C. (1995). De la mesure de la sensibilité temporelle aux modèles d'organisation temporelle: hypothèses et données sur l'acquisition des capacités temporelles auditives. L'Année Psychologique, 95, 555-569.

Drake, C., Jones, M. R., \& Baruch, C. (2000). The development of rhythmic attending in auditory sequences: attunement, referent period, focal attending. Cognition, 77, 251-288.

Fraisse, P. (1974). Psychologie du rythme. Paris: Presses Universitaires de France.

Gérard, C., \& Rosenfeld, M. (1995). Pratique musicale et régulations temporelles. L'Année Psychologique, 95, 571-591.

Gibbon, J., Malapani, C., Dale, C. L., \& Gallistel, C. R. (1997). Toward a neurobiology of temporal cognition: advances and challenges. Current Opinion in Neurobiology, 7, 170-184.

Harrington, D. L., Haaland, K. Y., \& Hermanowicz, N. (1998). Temporal processing in the basal ganglia. Neuropsychology, 12(1), 3-12.

Ivry, R. B., \& Keele, S. W. (1989). Timing functions of the cerebellum. Journal of Cognitive Neuroscience, 1(2), 136-152.

Ivry, R. B., \& Richardson, T. C. (2002). Temporal control and coordination: the multiple timer model. Brain and Cognition, 48, 117-132.

Jones, M. R. (1976). Time, our lost dimension: toward a new theory of perception, attention and memory. Psychological Review, 83, 323-355.

Jones, M. R., \& Boltz, M. (1989). Dynamic attending and responses to time. Psychological Review, 96, 459-491.

Lewkowicz, D. (2000). The development of intersensory temporal perception: an epigenetic systems/limitations view. Psychological Bulletin, 126 (2), 281-308.

Lewkowicz, D. (2003). Learning and discrimination of audiovisual events in human infants: the hierarchical relation between intersensory temporal synchrony and rhythmic pattern cues. Developmental Psychology, 39 (5), 795-804.

Pouthas, V., Provasi, J., \& Droit, S. (1996). Biobehavioral rhythms: development and role in early human ontogenesis. In J. T. Fraser \& M. P. Soulsby (Eds.), Dimensions of time and life-The study of time VIII (pp. 19-30): International universities press, inc.

Provasi, J. (1988). Capacités et apprentissage de régulations temporelles chez le nourrisson de l'activité de succion. Thèse, Université René Descartes, Paris.

Provasi, J., \& Bobin-Bègue, A. (2003). Spontaneous motor tempo and rhythmical synchronization in 21 /2- and 4-year-old children. International Journal of Behavioral Development, 27(3), 220-231.

Schmuckler, M. A. (1996). Visual-proprioceptive intermodal perception in infancy. Infant Behavior and Development, 19 (2), 221-232.

Thelen, E. (1979). Rhythmical stereotypies in normal human infants. Animal Behavior, 27, 699-715. 
Vanneste, S., Pouthas, V. \& Wearden, J.H. (2001). Temporal control of rhythmic performance: a comparison between young and old adults. Experimental Aging Research, 27, 83-102.

Wing, A. M., \& Kristofferson. (1973). The timing of interresponse intervals. Perception \& Psychophysics, 13 (3), 455.

Wing, A. M., \& Kristofferson, A. B. (1973). Response delays and the timing of discrete motor responses. Perception and Psychophysics, 14 (1), 5-12.

\section{NOTES}

1. A tempo is characterized by its Inter Onset Interval (IOI) value. For example, a $600 \mathrm{~ms}$ IOI tempo or a $600 \mathrm{~ms}$ tempo is an isochronous sequence where tones occurs every $600 \mathrm{~ms}$.

\section{ABSTRACTS}

Durations of less than one second are involved in all kinds of activities, such as music perception or rhythmical production tasks. It is therefore important to know how short durations are processed at a young age. This study aims to define discrimination performance between 3- and 4-year-olds (tempo discrimination). The first 4 years of life are essential for cognitive development and there is little data concerning those years due to the extreme difficulty of finding methods to assess children's timing performances. Moreover, the results yielded by previous experiments are controversial. Our data revealed that discrimination performances improve significantly within only a few months. The discrimination data from 3 and 4 year-olds are compared to those from older children. The overall results are discussed in a developmental model context.

Les durées de moins d'une seconde sont impliquées dans de nombreuses activités telles que la musique ou encore la production de rythmes. La question est de savoir comment se met en place ce traitement des durées courtes au cours des premières années de la vie. Plus spécifiquement, la question posée par cette étude est de déterminer les performances de discrimination temporelle entre 3 et 4 ans. En effet, l'enfant, au cours des 4 premières années, subit un développement cognitif important. Or, très peu de données sont disponibles pour cette période essentielle car il est extrêmement difficile de mettre au point des protocoles expérimentaux adaptés pour évaluer les performances des enfants. De plus, les données existantes sont contradictoires. Nos résultats montrent que les performances de discrimination s'améliorent significativement en seulement quelques mois. L'ensemble des résultats est discuté dans le cadre d'un modèle développemental.

\section{INDEX}

Keywords: children, Short Duration, Discrimination, Time Perception, Threshold, Auditory Tempo, Development 


\section{AUTHORS}

\section{ANNE BOBIN-BÈGUE}

Laboratoire de Psychobiologie du Développement, EPHE

41, rue Gay-Lussac 75005 Paris, France

Phone number:(33) 1441078 17, Fax:(33) 143268816

anne.begue@freesurf.fr

\section{JOËLLE PROVASI}

Laboratoire de Psychobiologie du Développement, EPHE (Paris, FRANCE) 